CAMBIOS EN LA

\section{ESTRATEGIA DIDÁCTICA}

DE EMPRESA DE

COMUNICACIÓN

\section{De la clase magistral a la}

\section{pregunta socrática}

\section{María José Pérez Serrano}

\section{Profesora de Empresa de Comunicación}

Facultad de Ciencias de la Comunicación. Universidad San Jorge. Autovía A-23 Zaragoza-Huesca, Km. 510. Telf. 976060100 - Email: mjperez@usj.es - http://www.usj.es

\section{Resumen}

El EEES implica una transformación radical en los planteamientos didácticos y metodológicos; es decir, requiere una permanente innovación en la praxis del aula rigurosamente fundamentada en pos de un incremento de la calidad educativa.

En estas páginas intentaremos ahondar en las bases de la docencia de uno de los segmentos conceptuales más importantes de las antes llamadas Ciencias de la Información, es decir, Empresa de Comunicación (EC). Esos fundamentos gravitarán sobre dos de los apartados más destacados de la secuencia didáctica tales como son las metodologías de enseñanza-aprendizaje y el sistema de evaluación,

\section{Palabras clave}

Empresa de Comunicación, Ciencias de la Comunicación, Espacio Europeo de Educación Superior (EEES), metodologías de enseñanza-aprendizaje, ECTS, evaluación Key Words

Media Business, Communication Sciences, European Higher Education Area (EHEA), teaching and learning methods, European Credit Transfer System (ECTS), assessment

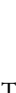

The EHEA means a radical transformation of teaching and methodological approaches, it is to say, it requires constant innovation in classroom practice rigorous-

ly grounded in pursuit of increased standards of educational quality. In these pages, we try to deepen the teaching bases in one of the most important conceptual segments formerly known as information science, that is to say, Media Business (MB). These fundamentals will gravitate on two of the most prominent points of the didactic sequence such as teachinglearning methodologies and assessment system, both included in the "quasi contract" pattern of the daily academic course represented by study guide. As a symptom of this, the subtitle suggests the two most important ends of teaching transformation. On the one hand, the lecture is the model of a university system very unidirectional, and, secondly, the

"Socratic" question symbolizes the interaction between student and teacher in the latter, with their questions, helps the student in his learning process and take it out of lack of knowledge in an area where traditionally the methodological foundation has focused on the teacher. 
incluidas ambas en la pauta "cuasi contractual" del decurso académico diario que representa la guía docente.

Como síntoma de ello, en el subtítulo se apuntan los dos extremos más importantes de la transformación docente. Por un lado, la clase magistral representa el paradigma de un sistema universitario de marcado ascendente unidireccional, y, por otro, la pregunta socrática simboliza la interacción entre el alumno y el profesor en la que éste, con sus cuestiones, ayuda al discente en su proceso de aprendizaje y lo saca de la ausencia de la conocimiento en un área en la que tradicionalmente el fundamento metodológico se ha centrado en el docente.

\section{Introducción}

En cualquier nivel educativo, las coordinadas pedagógicas tienen como premisa que el alumno es el sujeto de su educación. Por esta razón, partir de su situación académica; evitar vacíos y discontinuidad en la formación; promover el desarrollo de la capacidad de "aprender a aprender" para la firme asimilación de nociones; favorecer la autonomía personal mediante el dominio y aplicación sistemática de técnicas de estudio y trabajo intelectual, así como el empleo de las nuevas tecnologías, y lograr la construcción de aprendizajes sólidos y significativos, capaces de transferirse a otras materias, son principios fundamentales de la labor didáctica del docente.

La etapa formativa en la que nos centraremos está marcada por el modelo derivado de la Declaración de Bolonia, que busca revivir en la universidad aquello de lo que su semántica hacía gala: un modelo universal con una trascendental repercusión de las metodologías de enseñanza-aprendizaje. Y el alumno del que nos ocuparemos es el que cursa alguna de las ramas de las Ciencias de la Comunicación, que, con un currículum epistemológico necesariamente joven, demanda una concepción vanguardista y una práctica pegada a una realidad laboral cambiante.

En este contexto, el objeto de estas páginas se encuentra en reflejar algunas de las innovaciones docentes en la asignatura de Empresa de Comunicación, en concreto las relacionadas con los métodos de enseñanzaaprendizaje y el sistema de evaluación diseñado, teniendo como eje la integración del proceso evaluador en la aprehensión y utilización por el discente de competencias específicas vinculadas a esta materia. 


\section{Objetivos}

Precisada la finalidad, pasamos a desgranar algunos de los elementos claves que lo estructuran y componen. Hemos señalado que el objeto de esta empresa no es otro que presentar la combinación de metodologías de enseñanza-aprendizaje que se utilizan en una asignatura como la que nos ocupa, así como plantear un sistema de evaluación que tiene al alumno como principal protagonista, que se concibe y vertebra más como un medio de aprendizaje que como valoración de conocimientos, y se desarrolla a través de criterios multivariables debidamente esquematizados.

Esta interdependencia metodologíaevaluación se puede concretar en la siguiente tríada de objetivos:
- Analizar la pertinencia y viabilidad de las innovaciones docentes en una asignatura universitaria tradicionalmente de corte teórico y magistral.

- Aportar pautas ponderadamente útiles para que el alumno se implique en mayor media en el aprendizaje de Empresa de Comunicación.

- Ofrecer determinados sistemas de valoración para la evaluación continua en esta área, que ayuden a conocer el grado de consecución de las competencias genéricas y específicas refleja-das en la guía docente.

\section{Metodología}

Como síntesis metodológica de estas páginas, conviene señalar que, partiendo de la definición de didáctica como la:

disciplina de carácter práctico y normativo que tiene por objeto específico la técnica de la enseñanza, es decir, el conjunto sistemático de principios, normas, recursos y procedimientos específicos que todo profesor debe conocer y saber aplicar para orientar con seguridad a sus alumnos en el aprendizaje de las materias de los programas, teniendo en vista sus objetivos educativos son tres los pilares que sustentan nuestra reflexión: el diseño e implementación de métodos de enseñanza-aprendizaje más interactivos; la delimitación de tareas específicas acordes a esos presupuestos metodológicos, y la aplicación -como ejemplo- de consecuentes sistemas de evaluación, cada vez más complejos y poliédricos que aúnen teoría y práctica.

Para mostrar la progresión discursiva, escogemos el método de caso (Empresa de Comunicación) por dos razones. La primera, porque está positivamente ponderado en la nueva docencia universitaria; de este 
modo, aquí, método y objeto de estudio se interrelacionan, lo que puede hacer aún más interesante el acercamiento planteado. Y, segunda, porque el análisis del caso, según Walker, es "un examen de un ejemplo en acción", y nada mejor para completar los objetivos propuestos.

Conviene hacer una acotación en cuanto al título. Como precisión semántica debemos señalar que el término didáctica lleva implícito una parte de táctica, pero aquí se enfatiza en la estrategia como sinónimo del esfuerzo de diseño e implementación de pautas didácticas novedosas. El subtítulo, asimismo, plantea los extremos de un vector que no tienen por qué ser antagónicos, pero sí representan síntomas de un cambio de mentalidad en la labor del profesor.

El cuerpo del artículo se estructura en tres partes: en primer lugar, la contextualización de la materia objeto de estudio; en segundo, el análisis de su estrategia docente y, por último, las observaciones sobre la trascendencia en la estrategia didáctica.

\section{Empresa de Comunicación}

\subsection{Contexto y concepto}

Con el fin de entender cuál es la materia objeto de estudio diremos que se trata de un constructo teórico que emana del estudio sistematizado de los elementos, estructura, funciones y funcionamiento de las organizaciones cuyo objeto resulta ser la información en sentido amplio. En el contexto académico, se trata de un corpus teórico inserto en los estudios de Comunicación cuyas ramificaciones exigen un COnocimiento interdisciplinar de un mundo más que cambiante; por ello, la conceptualización de este tipo de empresas se apoya en tres ejes fundamentales: el derecho, la economía y, fundamentalmente, el periodismo. Desde este prisma, la ausencia de alguno de los tres soportes supondría un estudio incoherente, incompleto y carente de perspectiva global.
Esta asignatura permite, pues, plantear un marco conceptual del negocio de los mass media que ayuda a comprender la dinámica económica, la dirección y la gestión de las sociedades cuyo objeto es la información, en sus distintas formas y soportes, así como conocer los procesos de crecimiento externo, como paradigma de los actuales cambios en el mercado de la información. El acercamiento cognitivo, gnoseológico y práctico a este sector empresarial posibilita al estudiante, en un primer nivel, obtener la base conceptual imprescindible para otras materias como puede ser Estructura de la Comunicación y, al futuro periodista, integrarse con éxito en un entorno laboral complejo. 


\subsection{Sus competencias}

En cuanto a las competencias genéricas (marcadas por el Libro blanco de los títulos de grado en Comunicación, 2007) que le corresponden a esta materia son la capacidad de análisis y síntesis; la resolución de problemas; la capacidad de organización y planificación; el trabajo en equipo; la capacidad de aplicar conocimientos, y la capacidad de investigación.

Las competencias específicas de la titulación que incluye esta área giran en torno a los siguientes ítems: la capacidad de experimentar e innovar mediante el conocimiento y para incorporarse y adaptarse a un equipo profesional, haciendo compatibles, e incluso sinérgicos, los propios intereses particulares y los del proyecto colectivo en el que se insertan; la adaptación a los cambios; la capacidad para incorporarse la capacidad para asumir el liderazgo en proyectos que requieran recursos humanos y de cualquier otra naturaleza, gestionándolos eficientemente, asumiendo los principios de la responsabilidad social, y el cono- cimiento de la estructura, funcionamiento y gestión de la empresa de comunicación.

Por último, algunas de las competencias específicas que se han diseñado particularmente para Empresa de Comunicación son: entender la empresa de comunicación como una tipología dentro de los sectores económicos, señalando sus peculiaridades y semejanzas; analizar el marco jurídico de las sociedades dedicadas a la información; conformar y entender el entramado económico de la empresa informativa; conocer e interpretar el nuevo Plan General de Contabilidad de las empresas de comunicación, como herramienta de comunicación externa y situacional dentro del mercado global; asentar las bases de la dirección de medios de comunicación; configurar un plan de marketing periodístico diseñado desde una perspectiva global de la empresa comunicativa; conceptualizar la concentración de medios, sus causas y sus consecuencias, y analizar e interpretar procesos de crecimiento externo de las empresas de comunicación tanto españolas como extranjeras.

\section{Estrategia didáctica}

Si la estrategia es el arte de dirigir un asunto, corresponde en este epígrafe analizar cuáles son los caminos que conducen al alumno al conocimiento de esta materia y cómo se evalúan. Para ello es importante saber que la educación, en sus distintos niveles, se basa en los principios de la pla- nificación, que no son otros que fijar objetivos, establecer medios e instrumentos para su logro, estructurar la serie de tareas, analizar el proceso, sintetizar resultados y evaluar la eficacia entre el esfuerzo y los recursos aplicados, es decir, averiguar si el rendimiento final es adecuado. 
Estas líneas de actuación aparecen ya, como pauta de la didáctica universitaria, en la guía docente que, normalmente, se estructura en descripción, competencias, objetivos, requisitos, métodos, contenidos específicos y conexos, actividades de enseñanza-aprendizaje, controles, ponderación de variables (personales, sociales, institucionales), y evaluación de resulta-dos con sus secuelas valorativas, interpretativas y proyectivas respecto al alumno, al profesor y al propio sistema. En ello, la referencia estadística, al permitir la abstracción y extrapolación de datos, facilita la tarea evaluadora en el contexto dinámico y de movilidad estudiantil que subyace en la filosofía controlada por el BFUG (Bologna Follow Up Group).

\subsection{Metodologías de enseñanza-aprendizaje}

Las metodologías de enseñanza-aprendizaje de Empresa de Comunicación se descomponen en sesiones teóricas, prácticas $\mathrm{y}$ tutorías.

Los aspectos teóricos se desarrollan mediante exposiciones magistrales, de carácter analítico-sintético y basadas en el método deductivo, con apoyo de las TIC. Desde el primer momento, se fomenta el feed-back y la discusión socrática, en su triple secuencia de ironía, inducción y mayéutica, como puntos esenciales que aportan validez al método. El planteamiento del programa se entiende desde un conjunto coherente y lineal, en cuanto al proceso de adquisición de conocimiento, pero circular o globalizante en cuanto al objetivo final. Se asiste, así, al comienzo de la vida de la empresa dedicada a la información y se intenta, desde el principio, integrar al alumno en la comprensión de la comunicación como actividad comercial o mercantil.

Las sesiones prácticas se diseñan desde un planteamiento globalizador, adaptado a las necesidades de actualización constante y cercano a la realidad empresarial que esta asignatura exige; por ello, es necesario introducir en la praxis el aprendizaje cooperativo. En este marco se emplean metodologías como el trabajo en equipo, ya que el conocimiento se constata con la interacción, los alumnos buscan una meta común, y son evaluados por su participación solidaria y por el resultado conjunto; el método del caso, que se emplea desde una doble perspectiva: el planteamiento de situaciones concretas inventadas con finalidades pedagógicas específicas para fomentar el análisis, la toma de decisiones, y la propuesta de casos reales que sirvan para la aproximación a la realidad; y, también, para conseguir que el alumno aprehenda e interprete una situación coetánea con su período de estudios (esta labor se hace especialmente relevante en el análisis de procesos de concentración de medios, empleando el prisma interpretativo estudiado en la parte presencial); el trabajo individual a través de la realización de ejercicios, con una dificultad creciente, sobre las finanzas de la empresa, y otras 
metodologías como el ABP (Aprendizaje Basado en Problemas) para la captación, por ejemplo, del mecanismo del funcionamiento de las estructuras jurídicas empresariales.

Aunque lo virtual, según los criterios derivados de la moderna didáctica, resulta ser un espacio válido para el aprendizaje, enfatizamos aquí en el valor de la relación profesor-alumno como base del aprendizaje, difícilmente superable por ningún elemento técnico. Este énfasis se hace patente en el horario de tutorías, durante el cual el alumno puede preguntar dudas, ampliar bibliografía o solicitar aclaraciones sobre cualquier aspecto del temario, sin pretender -por supuesto- reemplazar el valor de la clase teórica. También son factibles tutorías grupales para la resolución de cuestiones relacionadas con los trabajos en equipos o para la supervisión de las tareas desarrolladas en este apartado. El uso del e-mail para el planteamiento de problemas o puntualizaciones es normal para cuestiones derivadas de los temas teóricoprácticos tratados en clase o en tutorías, pero inaceptable si se trata de suplir absentismos injustificados.

\subsection{El sistema de evaluación en EC}

En una clásica definición de evaluación, Lafourcade (1978) señalaba que es "la etapa del proceso educativo que tiene como finalidad comprobar, de manera sistemática, en qué medida se han logrado los obje- tivos propuestos" que se inserta en un “proceso sistemático, destinado a lograr cambios duraderos y positivos en la conducta de los sujetos”.

Más de treinta años después, el concepto sigue siendo válido ya que, con carácter general, el proceso evaluador continúa orientándose, y ahora con mayor difusión, predicamento y comentario, a aportar información puntual y objetiva del nivel cognitivo y de competencia alcanzado por el alumno con respecto a los elementos básicos de un área determinada; a ofrecer datos en cuanto al grado de adquisición de técnicas imprescindibles para desarrollarse en el ámbito universitario y, llegado el momento, que le faculten para el ejercicio profesional. Además, tiene carácter formativo; permite adoptar medidas de refuerzo o ampliación según el ritmo y resultados parciales, y facilita el diagnóstico y evaluación de la acción académica y del propio sistema educativo. Individualizada, cualitativa, continua y orientadora, la evaluación debería ser, al fin, un medio para conseguir una formación integral del discente en cualquier campo del conocimiento humano.

\section{a) Claves y pilares del sistema}

Como ya se ha señalado la traslación pragmática del objetivo de calidad del EEES a la universidad española pasa, desde nuestro punto de vista, por la excelencia en 
todos los procesos de la didáctica, en donde destaca sobremanera la aplicación de sistemas de evaluación idóneos para cada una las metodologías de enseñanzaaprendizaje.

Por eso, para la asignatura de Empresa de Comunicación de Periodismo de la Facultad de Ciencias de la Comunicación de la Universidad San Jorge se ha planteado para el curso académico 2009-2010 un sistema de evaluación, en ningún caso rígido y estático, sino flexible y adaptado, para la docencia universitaria que se encuentra fundamentado en cuatro pilares fundamentales: el primero se denomina "momentos clave y tratamiento", que contiene tres fases: una evaluación inicial, que detecta el punto de partida mediante diversas metodologías como puede ser una prueba; la lectura de un texto ad hoc a la materia y resumen oral del mismo; la redacción sobre un tema libre relacionado con el área de estudio para conocer intereses, léxico específico, sintáctico, etc.; una evaluación continua, progresiva o sumativa, que controla e informa sobre la actividad diaria del alumno en el proceso de enseñanzaaprendizaje, y una evaluación final, que sintetiza e informa acerca de la consecución de objetivos fijados y de competencias.

El segundo es el llamado "prismas evaluadores”, en el que la evaluación es observada desde diferentes puntos de vista, implica a los protagonistas del proceso de enseñanzaaprendizaje y obliga a una actitud empática por parte de todos ellos: el del alumno, que comprueba y valora su propio rendimiento respecto a los objetivos establecidos tanto en el sector de conocimientos específicos como en lo referente a actitudes, autonomía personal, dominio de técnicas de estudio y trabajo intelectual, asistencia e interés, capacidad de investigación, selección de contenidos, interrelaciones de conceptos, síntesis armónica, análisis e interpretación de resultados e integración socio-académica; el del profesor: que tiene en la evaluación un medio que permite observar y describir con la mayor precisión posible los aspectos cuantitativos y cualitativos de la estructura de la materia específica, de la secuencia de aprendizaje y del producto final del proceso, considerando las situaciones problemáticas y progresos derivados del uso de otros idiomas (Content and Language Integrated Learning -CLIL-) y la integración intercultural del estudiante, y el normativo, que entiende la evaluación como un mejoramiento continuo a través del conocimiento lo más exacto posible de todos los aspectos del proceso didáctico.

El tercero es la "función evaluadora". Obviamente, poner en práctica este sistema de evaluación implica que la labor del profesor no queda limitada a la mera exposición temática y corrección posterior; sino que, además, supone una constante relación con el alumno para facilitar una mutua corriente de comprensión que allane obstáculos, así como la previa, y gradual, programación de actividades y recursos (sobre todo 
vinculados al empleo de tecnologías digitales).

Y la cuarta son los "métodos de evaluación”. En ellos, la actividad pedagógica, en su dimensión programática y de didáctica de aula y de, según la estructura del nuevo crédito ECTS (European Credit Transfer System), de su trabajo autónomo, tiende a que el alumno consiga el máximo rendimiento que sea capaz y a que sea valorado de la manera más fiable y objetiva posible.

En esta línea, algunos de los instrumentos básicos de evaluación que, por ser el más claro exponente de la participación directa de los alumnos en el sistema, son objeto de atención preferente por parte del profesor son las escalas de valoración para el control de metodologías de enseñanza-aprendizaje como el rol-playing, las tutorías individuales y grupales y el trabajo autónomo; controles periódicos a través de pruebas objetivas; trabajos individuales; trabajos en equipo con exposiciones orales y con un sistema de auto y co-evaluación; pruebas escritas finales en forma de cuestionarios de res-puesta libre y solución de problemas.

\section{b) Aplicación práctica}

Este sistema se utiliza, en estos momentos, en la asignatura de Empresa de Comunicación, en cuya docencia se ha apostado por la innovación, y por un más que probado esfuerzo del profesor para conseguir integrar teoría y praxis para, así, lograr que los estudiantes adquieran capacidades de autorregulación de su propio proceso de aprendizaje. Lo que se pone de manifiesto en la siguiente tabla.

Gráfico $n^{o}$ 1: Instrumentos de evaluación y obtención de la nota final

\begin{tabular}{|c|c|c|c|}
\hline & Instrumento de evaluación & \multicolumn{2}{|c|}{$\%$ de la nota } \\
\hline & 1. Escalas de valoración & \multicolumn{2}{|c|}{$5 \%$} \\
\hline & 2. Prueba escrita intermedia & \multicolumn{2}{|c|}{$3 \%$} \\
\hline \multicolumn{2}{|r|}{ 3. Ejercicio / trabajo individual sobre lecturas } & \multicolumn{2}{|c|}{$3 \%$} \\
\hline \multirow{4}{*}{ 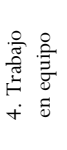 } & 4.1. Aportación individual al trabajo & $5 \%$ & \multirow{4}{*}{$25 \%$} \\
\hline & 4.2. Resultado conjunto del trabajo & $14 \%$ & \\
\hline & 4.3. Exposición (voluntaria) y defensa del trabajo en grupo & $3 \%$ & \\
\hline & 4.4. Prueba sobre trabajo en grupo & $3 \%$ & \\
\hline \multirow{2}{*}{ in } & 5.1. Resolución de ejercicios individuales & $8 \%$ & \multirow{2}{*}{$9 \%$} \\
\hline & 5.2. Trabajo voluntario sobre un caso de concentración & $1 \%$ & \\
\hline \multicolumn{2}{|r|}{ 6. Prueba escrita final } & \multicolumn{2}{|c|}{$55 \%$} \\
\hline & & \multicolumn{2}{|c|}{$100 \%$} \\
\hline
\end{tabular}

Fuente: Guía docente de Empresa de Comunicación. Curso 2009-2010 
En la evaluación de la "prueba escrita intermedia” (punto 2 de la tabla), desarrollada en $3^{\circ}$ de Periodismo - Grupo A, que constó de 60 ítems y una participación de 24 alumnos, se obtuvieron estos datos estadísticos: media 32,5 puntos; mediana 34,5 puntos, moda 40 puntos, y desviación estándar de 12,3 puntos. En la tabulación, se asignó al alumno con mayor puntuación el número 1 y el número 24 para el de menor. Ambos representan el rendimiento óptimo $(+2,058)$ e ínfimo $(1,991)$ de la serie, por lo que en la campana de Gauss ocuparían el punto inicial y el final. Analizando el rendimiento de cada alumno, se observa lo siguiente: el primero duplica el rendimiento normal (uno) y su esfuerzo merece mención honorífica; los tres siguientes sobresalen; otros cuatro estarían en línea notable; cuatro más se situarían en tono medio positivo; cinco podrían estimarse suficiente, pero negativo; y los siete finales denotan un rendimiento bastante inferior al mínimo, siendo pésimo el de los cuatro últimos.

Estos datos implican una reflexión sobre circunstancias del alumnado, condicionantes del sistema, metodología, recursos, etc., y demuestran que la evaluación, siguiendo estos parámetros, permite un conocimiento más fiable de la realidad académica y hace posible ratificar o rectificar proyectos y líneas de acción (Pérez Serrano, Pérez Camacho, 2009).

Otra consecuencia que se puede obtener de la aplicación de este sistema de evaluación es que ha habido un alto grado de coincidencia entre la percepción del profesor $y$ los estudiantes. Al ser cuestionados por su impresión, en pregunta abierta, entre un 65 y un $70 \%$ han considerado que el método ha sido complicado de asimilar en un primer momento, pero que, en cuanto se ha cogido el ritmo, les ha ayudado a programarse y a avanzar; que el trabajo en equipo supone esfuerzo añadido (aunque cada uno pudo escoger a sus otros cuatro compañeros de viaje), pero que ha tenido recompensa; que la didáctica -incluso- les ha ayudado a adquirir herramientas, recursos y modelos para la investigación científica que les serán útiles en un futuro. En cuanto al docente, el control final de todo el proceso le ha permitido concluir que no hay grandes disonancias entre las notas de auto-evaluación consignadas en la tabla evaluadora creada al efecto y las asignadas por éste, y que, aunque el esfuerzo profesional se incrementa exponencialmente, el resultado vale la pena. 


\section{Repercusión del sistema en la didáctica}

Desde la perspectiva planteada, la evaluación se integra en el proceso educativo convirtiéndose en un medio constante de diagnóstico y aprendizaje personal y significativo.

No se ha de hablar de evaluación aisladamente, por considerarla sólo la parte final del proceso, sino que se trata de una concepción de la educación en su integridad en la que la valoración está al servicio del aprendizaje y no éste supeditado a aquélla; es decir, el alumno se evalúa para aprender y no aprende para evaluarse, en línea con los principios de autonomía pedagógica universitaria y de los criterios de movilidad y validez de estudios derivados del EEES. Además, permite diagnosticar el propio sistema y adecuar los planteamientos teóricos a la realidad concreta.

Con esta premisa, la evaluación en Empresa de Comunicación no constituye el fin del proceso, sino que es parte esencial del mismo, pues, en contraposición con el concepto genérico de evaluación, como relación abstracta realizada en función de unos objetivos y contenidos predeterminados con referencia a un sujeto medio, aquí se considera algo concreto y centrado en la capacidad real del propio alumno. Interesan los conocimientos, pero interesa aún más que el aprendizaje no quede circunscrito al simple aspecto nocional, ya que éste se contempla como síntesis globalizadora y proyección de una personalidad cuasi profesional - con lo que esto implicaque se ha ido forjando a través de la participación activa, crítica y consciente del estudiante en su discurrir universitario.

Este sistema -más de trabajo que de evaluación- requiere una adecuada sistematización, y, aunque aparentemente la labor docente quede diluida por la decisiva participación del alumno, la realidad es que el profesor ha de coordinar y tutelar todo el proceso integrándose en el mismo y ejerciendo un minucioso control. Los referentes estadísticos, de compleja concreción cuando se refieren a trabajos de investigación, en los que las coordenadas esenciales han de quedar perfectamente acotadas para obviar subjetividades, así como los esquemas y tablas anexos son prueba fehaciente del rigor que debe presidir esta labor.

Así, la evaluación se convierte en una actividad constante que nos permite un conocimiento de cada alumno, del funcionamiento de la clase y de la propia estructura universitaria, y cumple una doble función: para el alumno, es una forma de aprender a la vez que de conocer por sí mismo su propio proceso de aprendizaje; es decir, su realidad individual y contextualizada; para el profesor, constituye una elocuente forma de enseñar a aprender y un instrumento de constatar resultados, tanto en el plano profesional como referido al sistema $y$ entorno institucional. 


\section{Conclusiones}

Los correlatos se pueden establecer en base a la siguiente triada:

1. La evaluación forma parte esencial de las metodologías de enseñanzaaprendizaje en una asignatura como Empresa de Comunicación.

2. El ideal es una forma flexible de evaluación en la que los alumnos se integren en el sistema, pero hay que ser consciente de la intrínseca dificultad que esto supone por lo que la función evaluadora del profesor es ineludible e impone un suplementario trabajo de control para armonizar los criterios de los alumnos con las exigencias específicas del área o asignatura de estudio.
3. Los cambios a los que asistimos no significan que las nuevas tendencias tengan que sustituir por completo al concepto de docencia que tradicionalmente ha funcionado a lo largo de la historia. Al aula, sea del nivel que sea, se va a trabajar y el estudiante debe ser consciente, y partícipe, de que la suya, como cualquier actividad laboral, implica esfuerzo. Además, es pertinente tener en cuenta que el profesor siempre es el guía y conductor de ese je y utiliza las nuevas estrategias y herramientas en función de los vos buscados, y que, en este caso, no desdeña la tradición docente, sino que la adapta a los nuevos retos de la universidad del siglo XXI.

\section{Referencias}

Agencia Nacional de Evaluación de la Calidad y Acreditación (2007). Libro blanco de los títulos de grado en Comunicación, 24 de septiembre de 2009 , www.aneca.es.

Lafourcade, P.D. (1978). Evaluación de los aprendizajes. Madrid: Cincel.

Pérez Serrano, M.J. (2009). Guía docente de Empresa de Comunicación. Curso 2009-2010, Zaragoza: USJ.
Pérez Serrano, M.J.; Pérez Camacho, J. (2009). "El sistema de evaluación como eje innovador en el proceso de enseñanza-aprendizaje del EEES”. En A. Medina Rivilla (Dir.), InnovaDOC 2009. Madrid: Pearson Educación.

Cita de este artículo

PÉREZ, M.J. (2009) Cambios en la estrategia didáctica de Empresa de Comunicación. De la clase magistral a la pregunta socrática Revista Icono14 [en línea] 25 de Octubre de 2009, № 14. pp. 149-160. Recuperado

(Fecha de acceso), de http://www.icono14.net 